\title{
Fatigue behaviour of AA6061-T6 alloys in the corrosive environment
}

\author{
Ngoc Vu Nguyen ${ }^{1}$, and Peifeng $\mathrm{Li}^{2, *}$ \\ ${ }^{1}$ School of Mechanical and Aerospace Engineering, Nanyang Technological University, Singapore \\ ${ }^{2}$ School of Engineering, University of Glasgow, Glasgow G12 8QQ, UK
}

\begin{abstract}
The combined effects of corrosion and fatigue are known to be hazardous threats to structural integrity of aluminium alloys that are being extensively used in marine applications. This work investigated the fatigue crack initiation and growth behaviour of AA6061-T6 alloys in $3.5 \mathrm{wt} \%$ $\mathrm{NaCl}$ simulated seawater using scanning electron microscope and electron backscatter diffraction characterisation techniques. It was found that the fatigue resistance of AA6061-T6 is drastically downgraded when subjected to the corrosive environment of $3.5 \mathrm{wt} \% \mathrm{NaCl}$ solution. High stress concentration at both sides of a pit mouth in conjunction with attacked grain boundaries facilitates fatigue crack nucleation, while the presence of hydrogen formed by corrosion reactions causes crack tip embrittlement and thus increases crack growth rate. Fractographic analysis reveals that there is a change in fatigue crack growth mechanism of AA6061-T6 alloys tested in the $\mathrm{NaCl}$ solution. At short crack length, the crack develops transgranularly along crystallographic planes due to hydrogen-enhanced decohesion process. Further crack growth is dominated by adsorption induced dislocation emission process, resulting in the mixed mode of intergranular and transgranular crack growth.
\end{abstract}

\section{Introduction}

Corrosion fatigue is the response of a material to alternating stresses in a corrosive environment by the initiation and propagation of cracks. It has been known as a dominant factor on the reduction of service life of marine structures and other equipment used in the offshore environment. Pitting corrosion combined with fatigue is the complex phenomenon involving various processes and mechanisms [1-4]. The pitting process causes local discontinuities on the surface of a structure and enhances the nucleation as well as propagation processes of small fatigue cracks. Due to the presence of these local discontinuities, short cracks can initiate and develop even below the threshold range of stress intensity factor determined for long crack growth [5]. In a corrosive environment, hydrogen is generated by cathodic corrosion reactions and then adsorbed at crack tips. There is broad consensus that degradation of crack growth resistance is caused by crack tip embrittlement due to the presence of hydrogen species.

Of the many proposed mechanisms, three most widely accepted interstitial mechanisms are: hydrogenenhanced localised plasticity (HELP), hydrogenenhanced decohesion (HEDE) and adsorption-induced dislocation emission (AIDE). The HELP mechanism is supported by the observations that solute hydrogen facilitates dislocation motion ahead of crack tips by forming an enhanced plastic zone size [6, 7]. The fracture process exhibits plasticity phenomena such as reduction of material yield strength and micro-void coalescences rather than embrittlement. However, this mechanism is only valid in a certain range of temperatures and strain rates. Contradictory results have been observed at elevated temperatures and higher strain rates. The second mechanism HEDE assumes that hydrogen adsorption weakens interatomic bonds at crack tips, resulting in decohesion (tensile separation) along cleavage planes or grain boundaries [8-10]. Therefore, the fracture surface may exhibit either brittle intergranular facets or transgranular cleavage. This mechanism is supported by brittle characteristics similarly observed in solid-metal induced embrittlement and metal-vapour induced embrittlement where decohesion occurs in the absence of considerable local deformation. The third mechanism AIDE proposed by Lynch [11] is based on fractographic observations such as extensive localised slips or nanoscopic dimples, indicating that crack growth occurs by localised slip or void coalescence processes. The AIDE mechanism assumes that adsorption of embrittling species weakens interatomic bonds and thereby enhances dislocation movements ahead of crack tips.

Fundamental understanding of hydrogen embrittlement mechanisms has been appreciably achieved thanks to advances in improved theoretic tools as well as high resolution experimental instruments. However, the embrittlement mechanisms and kinetics

\footnotetext{
Corresponding author: peifeng.li@glasgow.ac.uk
} 
involved are not fully resolved and are still controversial disputes as they cannot be generally applied to all materials in a particular environment such as the combination of corrosion and cyclic stresses.

This study investigated the effect of pitting corrosion on fatigue behaviour of AA6061-T6 alloys with the aim of having a better understanding of fatigue crack initiation and propagation mechanisms of aluminium alloys in a corrosive environment.

\section{Experimental procedure}

\subsection{Materials and specimens}

The material investigated in this study was aluminium alloys AA6061-T6 in the form of rolled sheet with a thickness of $4.06 \mathrm{~mm}$. Dog bone fatigue specimens were fabricated in the rolling-transverse orientations with the rolling direction for cyclic loading. The dimension of the specimens was designed according to ASTM E466 standard requirements (Fig. 1). Prior to fatigue testing, the specimen surfaces were ground with the subsequent use of 800 and 1200 grit silicon carbide paper to ensure the surface roughness $R_{\mathrm{a}}$ less than $0.15 \mu \mathrm{m}$.

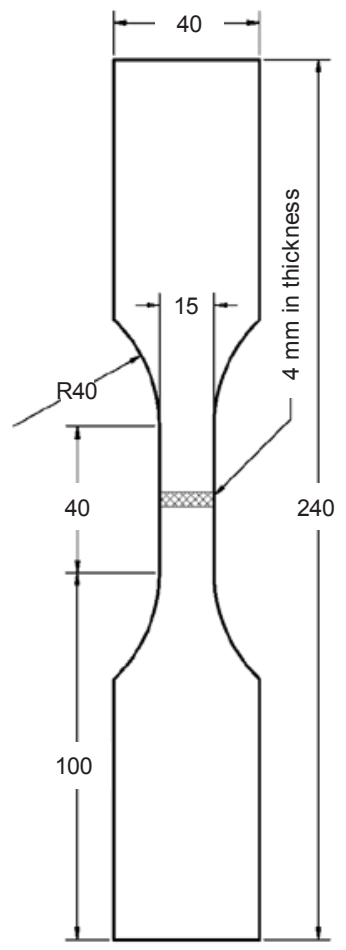

Fig. 1. Geometry and dimensions of the fatigue specimen. (unit: $\mathrm{mm}$ )

\subsection{Corrosion fatigue testing}

Fatigue experiments were conducted on the specimens under fully reversed tension-compression loading (stress ratio $R=-1$ ) at room temperature with the load frequency $f=5 \mathrm{~Hz}$ in an MTS 810 (MTS Systems Corp., USA) servo-hydraulic universal testing machine. At each stress level, at least three tests were repeated and the average number of cycles to failure was calculated to determine the $S-N$ curve (the cyclic stress amplitude versus the number of cycles to failure) of the material. The fatigue tests were performed in a corrosive environment as well as in the air atmosphere to analyse the effect of corrosion on fatigue behaviour of aluminium alloys.

Fig. 2 shows the overall setup of fatigue testing of an aluminium alloy specimen in an environmental chamber in the MTS 810 machine. The fatigue specimen especially the gauge portion was immersed into the environmental chamber that consisted of $3.5 \mathrm{wt} \%$ sodium chloride $(\mathrm{NaCl})$ simulated seawater. The $\mathrm{NaCl}$ solution was circulated through the environmental chamber by a peristaltic pump to provide continuous aeration and replenishment of the corrosive solution. The $\mathrm{pH}$ of this synthetic seawater solution was adjusted to 8.2 , and the specimens were exposed to the solution environment for one hour before applying the cyclic load.

Immediately after the corrosion fatigue failure, the specimens were taken out of the chamber and cleaned to preclude corrosion processes from obscuring fine details of the fracture surfaces. Fatigue tests of some specimens in the corrosive environment were interrupted prior to failure and examined by optical microscope to identify critical cracks. Once a critical fatigue crack was identified, the specimen portion containing this crack was shortly cut, polished by the colloidal silica $0.04 \mu \mathrm{m}$ suspension for two minutes and then etched by Keller's reagent for 30 seconds to reveal the microstructure for examination. Fracture features of these specimens were examined in a field emission scanning electron microscope (FE-SEM) JEOL 7600 (JEOL Ltd., Japan) equipped with an electron backscatter diffraction (EBSD) detector. 


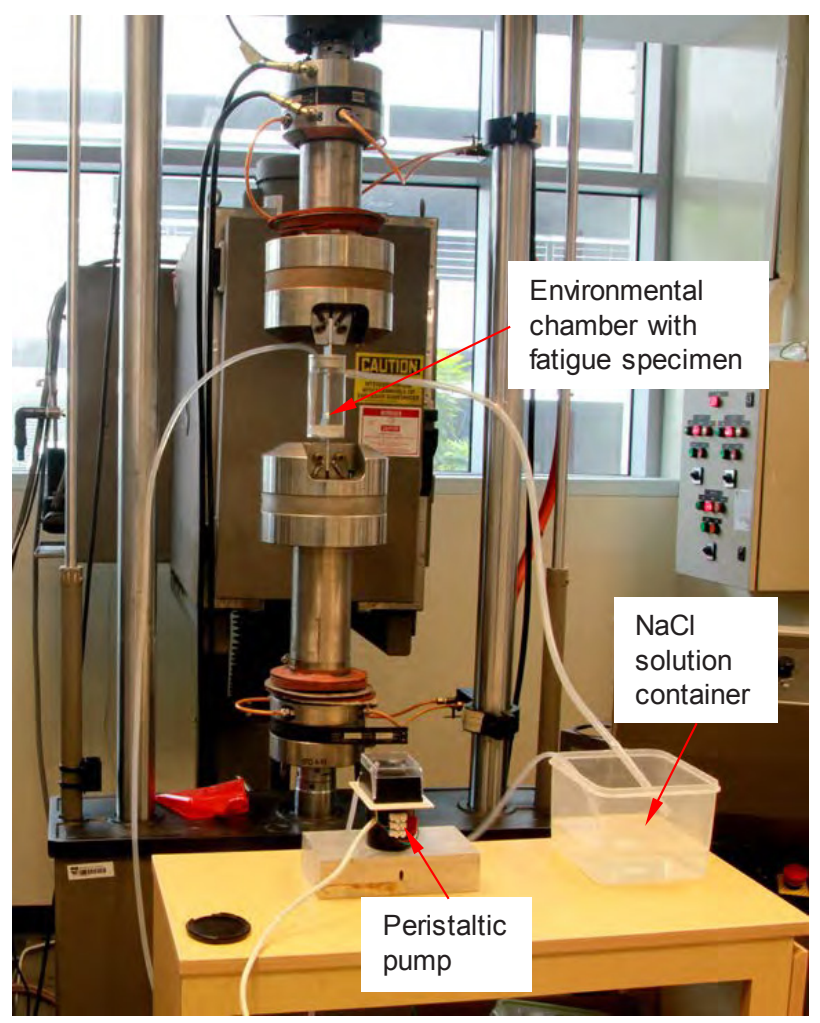

Fig. 2. Corrosion fatigue testing setup in the MTS 810 servohydraulic machine.

\section{Results and discussion}

\subsection{Fatigue $S-N$ curves}

The fatigue lives of AA6061-T6 alloy specimens tested in the air atmosphere and in the $3.5 \mathrm{wt} \% \mathrm{NaCl}$ solution were measured at different stress levels. Fig. 3 illustrates the plot of the average number of cycles to failure $\left(N_{\mathrm{f}}\right)$ versus the applied cyclic stress amplitude $\left(\sigma_{\mathrm{a}}\right)$ on a logarithmic scale. As aluminium does not exhibit a typical fatigue endurance limit, there is a continuous reduction of fatigue life as the applied stress increases. The relation between the fatigue life and the stress amplitude can be quantitatively described using a power equation:

$$
\sigma_{\mathrm{a}} N_{\mathrm{f}}^{\mathrm{m}}=C
$$

where $m$ and $C$ are the material constants determined from the fatigue experiments. The $S-N$ curves (Fig. 3) were fitted to estimate the constants $m=0.1018$ and $C=$ $580 \mathrm{MPa}$ for the specimens tested in the air while $m=$ 0.2538 and $C=2357 \mathrm{MPa}$ for the specimens tested in the $3.5 \mathrm{wt} \% \mathrm{NaCl}$ solution.

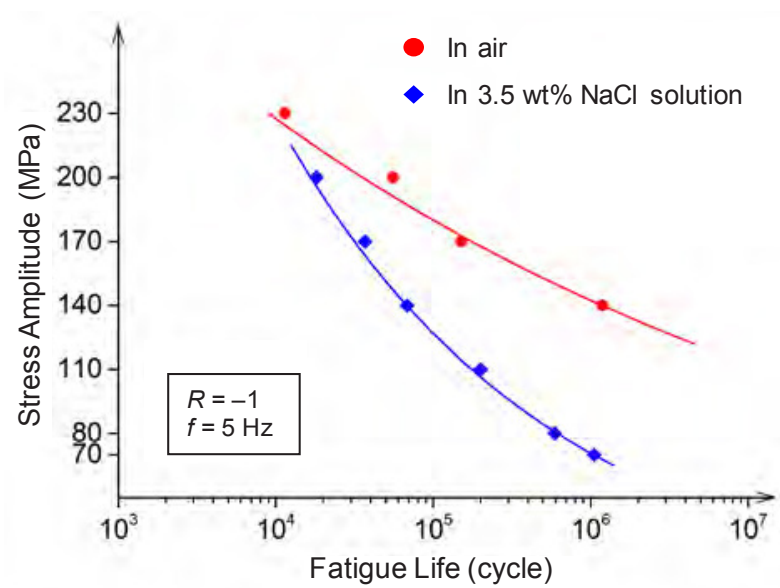

Fig. 3. Fatigue $S-N$ curves of AA6061-T6 alloys tested in the air atmosphere and in the corrosive environment of $3.5 \mathrm{wt} \%$ $\mathrm{NaCl}$ solution.

The fatigue resistance of AA6061-T6 alloys is significantly downgraded in the $\mathrm{NaCl}$ solution. For the fatigue life of more than one million cycles, the allowable fatigue strength in the $3.5 \mathrm{wt} \% \mathrm{NaCl}$ solution decreases by approximately $80 \mathrm{MPa}$ compared to the strength in the air atmosphere. At a higher stress amplitude of approximately $140 \mathrm{MPa}$, specimens subjected to fatigue loading in the air can sustain more than one million cycles. The damage $\left(N_{\mathrm{f}, \mathrm{NaCl}} / N_{\mathrm{f} \text {,air }}\right)$ at a specific applied stress is defined as the ratio of the average fatigue life tested in the $\mathrm{NaCl}$ solution $\left(N_{\mathrm{f}, \mathrm{NaCl}}\right)$ to that tested in the air $\left(N_{\mathrm{f} \text {,air }}\right)$. The damage ratio at three different stress levels $200 \mathrm{MPa}, 170 \mathrm{MPa}$ and $140 \mathrm{MPa}$ were calculated to be $3.06,4.08$ and 17.38 respectively. The increase of fatigue damage ratio in association with reduced stress amplitude indicates that the effect of corrosion environment on fatigue behaviour is more significant at lower stress amplitude as there would be more time for corrosion reactions and hydrogen embrittlement processes to occur in materials.

\subsection{Fractographic observations}

The effect of corrosion environment on fatigue crack initiation and propagation was elucidated using the SEM examiniations on the fracture features. Fig. 4 shows the fracture surface of a fatigue specimen failed in the $3.5 \mathrm{wt} \% \mathrm{NaCl}$ solution environment. Fatigue cracks nucleate from both sides of the pit mouth forming multiple crack fronts. The crack initiation site is a typical crystallographic pit. As these crack fronts further propagate, they meet and merge with each other, leaving a tearing ridge on the fracture surface (Fig. 4(a)).

Two stages of short crack can be distinguished by the detailed fractographic features as shown in Fig. 4(b-d). In the vicinity of the crack origin, the fracture surface appears to be flat and crack propagates transgranularly along crystallographic planes, forming cleavage patterns (Fig. 4(b)). As the crack grows to a critical length, extensive localised slips and fatigue striations can be observed on the fracture surface instead of cleavage features (Fig. 4(c, d). The different fractographic features 
indicate that there is a change in growth mechanism of short fatigue cracks in aluminium alloy when subjected to a corrosive environment.

\subsection{Mechanisms of fatigue crack initiation and propagation}

The higher crack growth rate and the resultant reduction of fatigue life of aluminium alloys in the corrosive environment can be attributed to the presence of hydrogen formed by cathodic corrosion reactions. The mechanism of pitting formation is based on the dissolution of aluminium in the anode:

$$
\mathrm{Al} \rightarrow \mathrm{Al}^{3+}+3 \mathrm{e}^{-}
$$

and several cathodic reactions, which could be the oxygen reduction:

$$
\mathrm{O}_{2}+2 \mathrm{H}_{2} \mathrm{O}+4 \mathrm{e}^{-} \rightarrow 4 \mathrm{OH}^{-}
$$

\section{a}

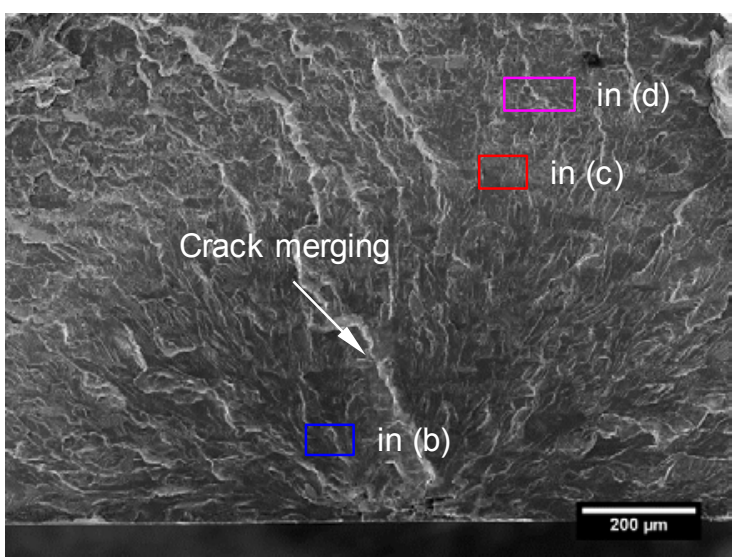

C

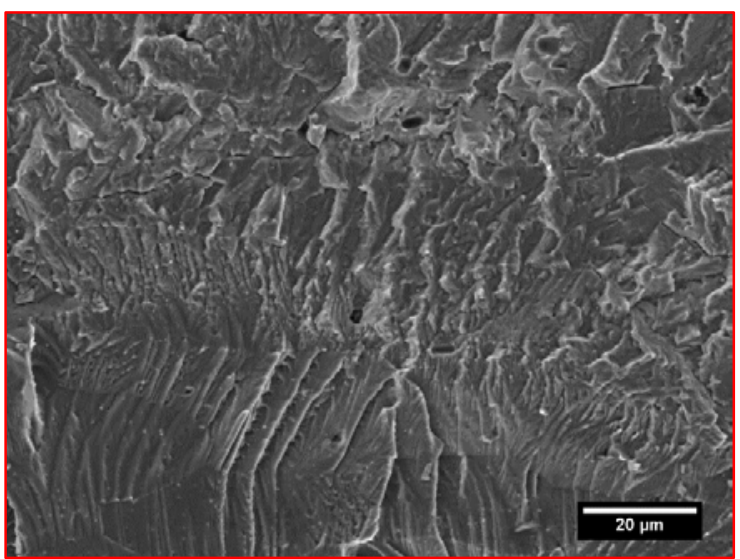

or hydrogen development in neutral or acidic environments:

$$
\begin{gathered}
2 \mathrm{H}^{+}+2 \mathrm{e}^{-} \rightarrow \mathrm{H}_{2} \\
2 \mathrm{H}_{2} \mathrm{O}+2 \mathrm{e}^{-} \rightarrow \mathrm{H}_{2}+2 \mathrm{OH}^{-}
\end{gathered}
$$

The presence of hydrogen causes crack tip embrittlement and escalates fatigue crack growth rate. Moreover, the cohesive bonding at crack tips can be weakened due to the high concentration of chloride in the simulated seawater. Fracture surface of corrosion fatigue specimens exhibits brittle characteristics in comparison with extensive ductile striations as in the case of fatigue samples tested in the air. It is generally accepted that hydrogen adsorption weakens interatomic bonds at crack tips and thus accelerates crack advancement.

b

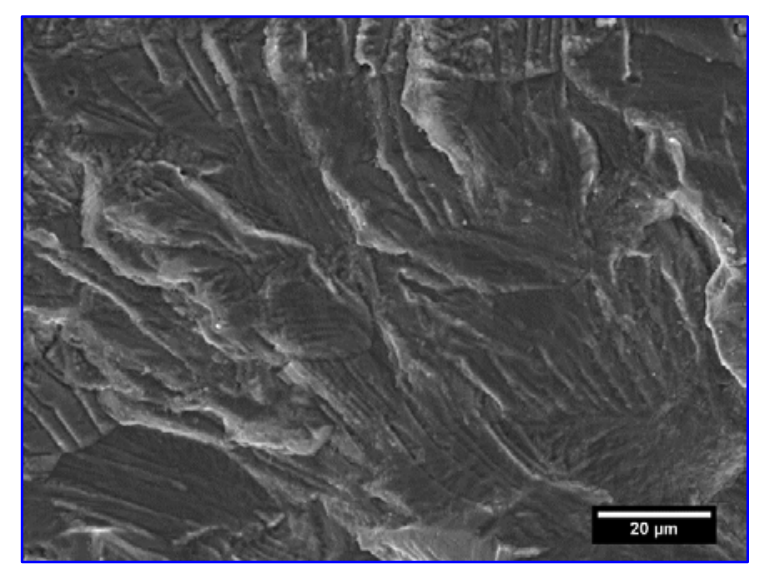

d

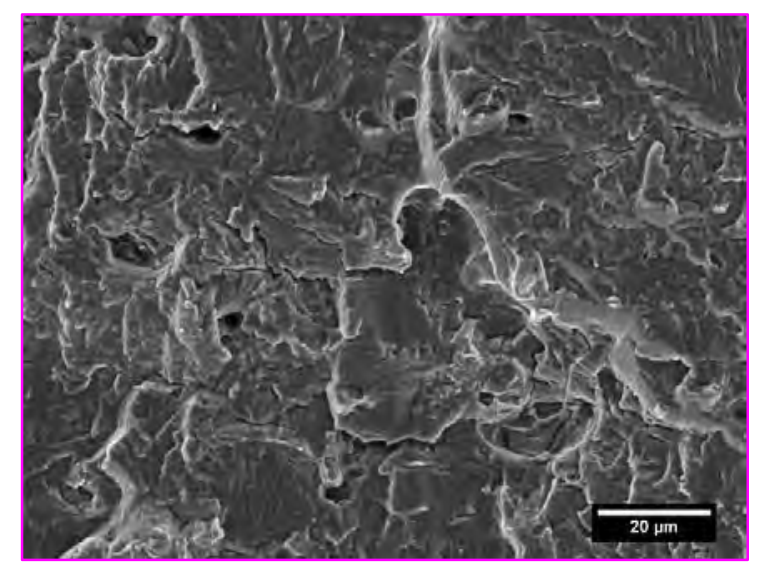

Fig. 4. SEM images of the fracture surface of a fatigue specimen tested in the $\mathrm{NaCl}$ solution: (a) overview, (b-d) details of selected fracture regions as indicated in (a).

However, the speed of hydrogen diffusion and adsorption could be responsible for the variation in crack growth mechanisms at different crack lengths. At short crack length where the crack growth rate is low, there is sufficient time for corrosion reactions to produce hydrogen and diffuse it to the crack front. Hydrogen is adsorbed at crack tips and weakens interatomic bonds such that decohesion occurs by tensile separation of interatomic bonds on low index crystallographic planes, i.e., the HEDE process. Capillary transport of hydrogen is limited as it requires a crack tip with significant radius. The plastic zone size ahead of the crack tip is 
relatively small at low stress intensity region, suggesting that the crack tip radius is too tiny to favour capillary flow.

As the crack grows further to a certain length corresponding to higher stress intensity, there would not be enough time for hydrogen diffusion at higher crack growth rate. Therefore, the crack tip is not embrittled yet, and most of dislocation activities caused by fatigue loading would produce crack tip blunting rather than cracking advancing because the plastic zone size is enhanced at higher stress intensity. This crack tip blunting process facilitates capillary transport of embrittling agents, hydrogen in this case, to the crack front. Capillary transport of hydrogen is dominant over surface diffusion at this stage of crack growth and thereby initiates the AIDE process. This process involves adsorption of embrittling species at crack tips and stimulating dislocation emission. This implies that most of dislocation induced by fatigue loading would advance fatigue crack on suitable slip systems. Therefore, extensive localised slips and fatigue striations occur at growing crack length as shown in Fig. 5.

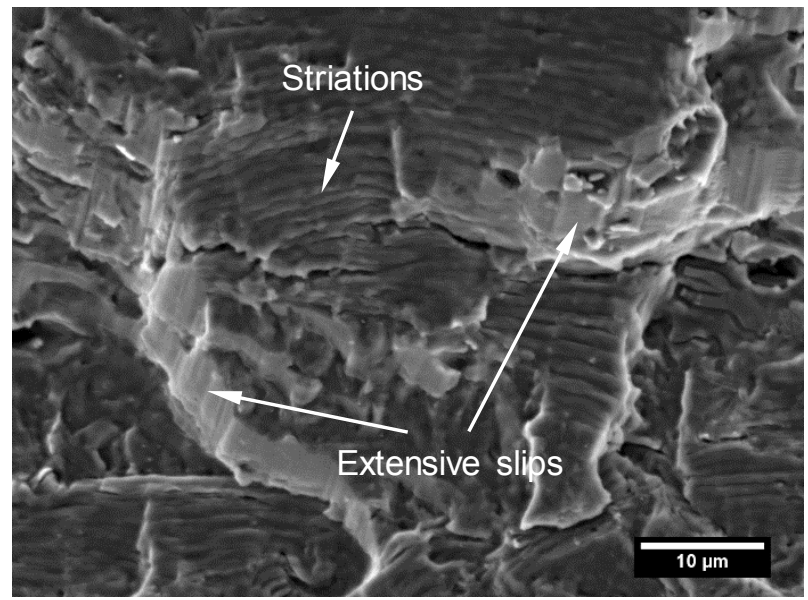

Fig. 5. SEM image of the fracture surface showing extensive localised slips and fatigue striations at growing crack length.

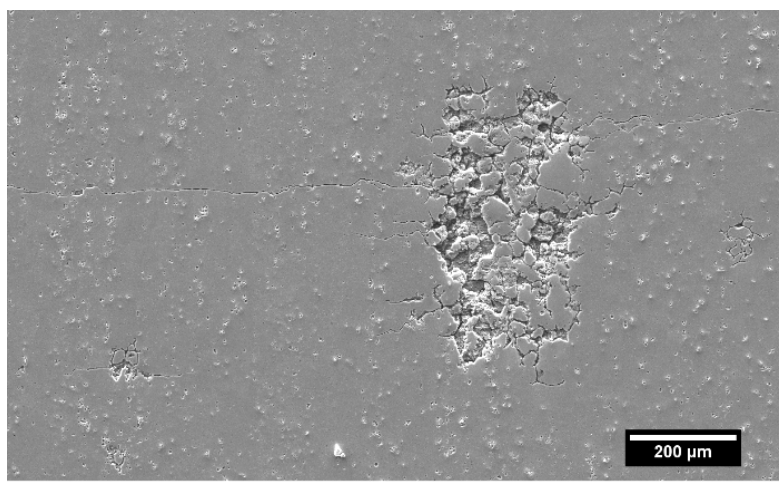

b
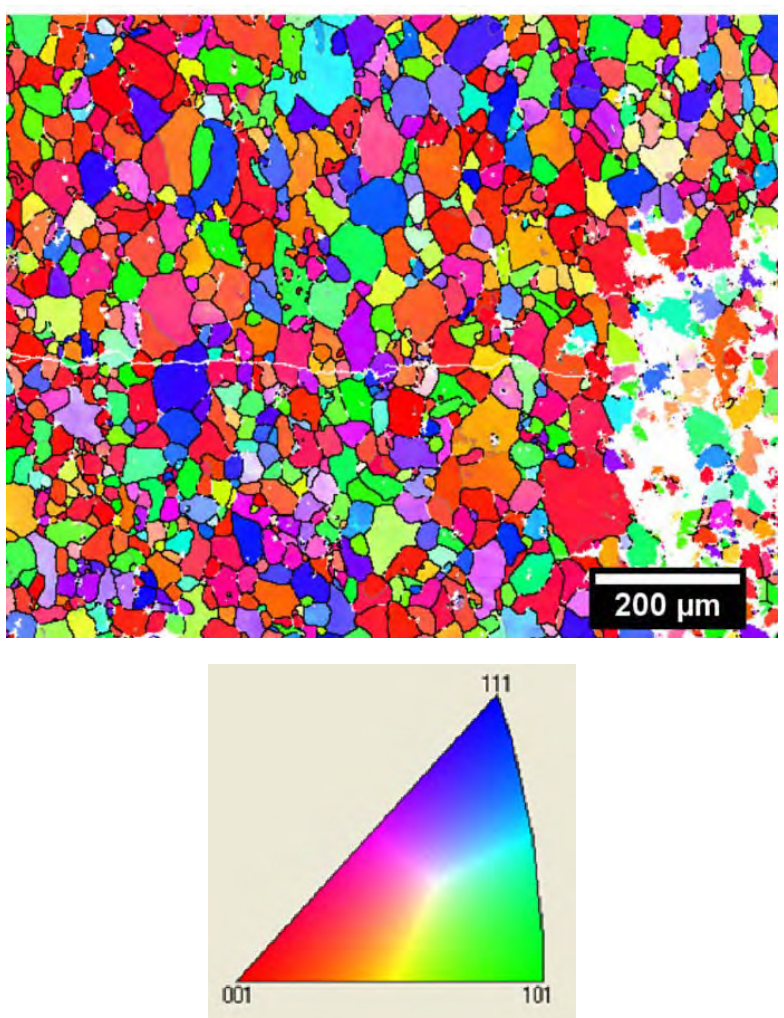

Fig. 6. (a) SEM image and (b) EBSD orientation map of a pit that initiates the fatigue crack.

The corrosion pit from which fatigue cracks initiate can be identified from the AA6061-T6 specimen subjected to interrupted fatigue in the corrosive environment (Fig. 6). The cracks initiate from both sides of the crystallographic pit mouth which are highest stress concentration sites [12]. Grain boundaries surrounding the corrosion pit are attacked due to anodic reactions of corrosion processes, which create a local acidic environment with high chloride concentration. This local acidic environment leads to a precipitate free zone, which facilitates intergranular corrosion at pit walls. Furthermore, fatigue loading can break down the protective aluminium hydroxide layer such that the aggressive environment can easily penetrate into the metal. High stress concentration at both sides of the pit mouth in conjunction with attacked grain boundaries favours fatigue crack nucleation and substantially 
reduces fatigue life as crack initiation can account for up to 80 percent of the total corrosion fatigue life. It should be noted that intergranular corrosion only occurs around the perimeter of crystallographic pits within the zone that local pitting potential is modified by corrosion reactions. Outside the corroded area, grain boundaries are not attacked, and the occasional occurrence of intergranular fatigue crack growth (Fig. 6(b)) does not involve intergranular corrosion but is associated with decohesion of grain boundaries due to the adsorption of hydrogen.

\section{Conclusions}

The fatigue performance of AA6061-T6 alloys is significantly downgraded in the $3.5 \mathrm{wt} \% \mathrm{NaCl}$ corrosive solution. Fatigue crack nucleation and propagation are affected by the corrosive environment. High stress concentration at both sides of the pit mouth in conjunction with attacked grain boundaries facilitates fatigue crack initiation. The presence of hydrogen formed by corrosion reactions causes crack tip embrittlement and intensifies the crack growth rate. The detailed fractographic analysis reveals that the crack growth can be distinguished by two stages. Near the crack origin, the crack propagates transgranularly along crystallographic planes due to the HEDE process. Further crack growth is dominated by the AIDE process, thus resulting in a mixed mode of intergranular and transgranular crack growth.

\section{References}

1. S.S. Singh, T.J. Stannard, X.H. Xiao, N. Chawla, In situ X-ray microtomography of stress corrosion cracking and corrosion fatigue in aluminum alloys, JOM, 69, 1404-1414 (2017).

2. M.N. Ilman, N.A. Triwibowo, A. Wahyudianto, M.R. Muslih, Environmentally assisted fatigue behaviour of stress relieved metal inert gas (MIG) AA5083 welds in $3.5 \% \mathrm{NaCl}$ solution, Int. J. Fatigue, 100, 285-295 (2017).

3. J.J. Schubbe, S.H. Bolstad, S. Reyes, Fatigue crack growth behavior of aerospace and ship-grade aluminum repaired with composite patches in a corrosive environment, Compos. Struct., 144, 44-56 (2016).

4. Z.Q. Hua, S.S. Wu, Tensile, fatigue, and corrosion fatigue behavior of high performance die cast aluminum alloy, J. Mater. Res., 30, 833-840 (2015).

5. J. Newman, E.P. Phillips, M. Swain, Fatigue-life prediction methodology using small-crack theory, Int. J. Fatigue, 21, 109-119, (1999).

6. H.K. Birnbaum, P. Sofronis, Hydrogen-enhanced localized plasticity - a mechanism for hydrogenrelated fracture, Mater. Sci. Eng. A, 176, 191-202 (1994).

7. I. Robertson, The effect of hydrogen on dislocation dynamics, Eng. Fract. Mech., 68, 671-692, (2001).
8. A.R. Troiano, The role of hydrogen and other interstitials in the mechanical behavior of metals, Trans. ASM, 52, 54-80 (1960).

9. R. Oriani, Hydrogen embrittlement of steels, Annu. Rev. Mater. Sci., 8, 327-357 (1978).

10. R. Oriani, A mechanistic theory of hydrogen embrittlement of steels, Berichte der Bunsengesellschaft für physikalische Chemie, 76, 848-857 (1972).

11. S. Lynch, Environmentally assisted cracking: overview of evidence for an adsorption-induced localised-slip process, Acta Metall., 36, 2639-2661 (1988).

12. Y.F. Huang, C. Wei, L.J. Chen, P.F. Li, Quantitative correlation between geometric parameters and stress concentration of corrosion pits, Eng. Fail. Anal., 44, 168-178 (2014). 